St andar di zat i on of net ai odobenzyl guani di ne heart to medi ast i num rat i o using a cal i br at i on phant om effects of correction on nor mal dat abases and a mil ti cent re st udy

\begin{tabular}{|c|c|}
\hline 著者 & $\begin{array}{l}\text { Nakaj i na Keni chi, Okuda Koi chi, Nat suo Shi nro, } \\
\text { Yoshi ta M t suhi ro, Taki Juni chi, Yamada } \\
\text { Nasahi to, K nuya Sei go }\end{array}$ \\
\hline $\begin{array}{l}\text { j our nal or } \\
\text { publ i cat i on } \mathrm{titl} \text { e }\end{array}$ & $\begin{array}{l}\text { Eur opean Jour nal of Nucl ear Medi ci ne and } \\
\text { Mbl ecul ar I magi ng }\end{array}$ \\
\hline vol une & 39 \\
\hline number & 1 \\
\hline page $r$ ange & 113119 \\
\hline year & 2012-01- 01 \\
\hline URL & ht t p: //hdl . handl e. net /2297/29562 \\
\hline
\end{tabular}




\title{
Standardization of metaiodobenzylguanidine heart-to- mediastinum ratio using a calibration phantom: Effects of correction on normal databases and a multi-center study
}

\author{
Kenichi Nakajima (I), Koichi Okuda (I), Shinro Matsuo (I), \\ Mitsuhiro Yoshita (2), Junichi Taki (I), Masahito Yamada (2), \\ Seigo Kinuya (I) \\ I. Department of Nuclear Medicine, Kanazawa University Hospital, Kanazawa, Japan \\ 2. Department of Neurology, Kanazawa University Hospital, Kanazawa, Japan
}

\section{Correspondence}

Kenichi Nakajima, MD, PhD

Department of Nuclear Medicine, Kanazawa University Hospital, I3-I Kanazawa, 920-864I, Japan, E mail: nakajima@med.kanazawa-u.ac.jp

\section{Footnotes}

Participating physicians and technologists in a multi-center MIBG standardization project in Japan: Kenichi Nakajima (Chief Investigator, Kanazawa University), Hiroshi Fukuda, Hayato Odagiri (Tohoku University, Sendai), Hidetoshi Morii (Sunagawa City Hospital, Sunagawa), Hiroshi Yokota, Masao Tamura (Tsukuba University Hospital,Tsukuba), Mana Yoshimura, Kenji Uchida, Kunihiko Hirayama (Tokyo Medical University Hospital,Tokyo), Satoru Suzuki, Yumiko Okuyama, Satoshi Kimura, Kenichi Sato (Juntendo University, Tokyo), Kengo Ito, Takashi Kato, Masato Saito (National Center of Geriatrics and Gerontology, Obu), Hidenao Miyoshi, Shigeru Moriyama (Tottori University, Tottori), Shuichi Sugino (Okayama Kyokuto Hospital, Okayama), Chio Okuyama, Takeshi Nii (Kyoto Prefectural University Hospital, Kyoto), Shinro Matsuo, Koichi Okuda, Junichi Taki (Kanazawa University, Kanazawa), Yumiko Kirihara, Takehiro Ishikawa (Fujifilm RI Pharma, Co. Ltd)

\section{Abstract}

Purpose. This study was performed to demonstrate that the results obtained with a calibration phantom could be used as a tool for standardizing measurement of heart-tomediastinum (H/M) ratio in cardiac metaiodobenzylguanidine (MIBG) imaging.

Method. Images of the phantom containing ${ }^{123}$ I-MIBG were acquired on the cameras in 10 hospitals (I I camera types) to determine the relationship between $\mathrm{H} / \mathrm{M}$ ratios using different collimators: low energy (LE) and medium-energy (ME)/low-medium-energy (LME) collimators. The effect of standardization to the ME-comparable $\mathrm{H} / \mathrm{M}$ ratio was examined in two settings: a Japanese standard MIBG database $(n=62)$ and multi-center studies $(n=49)$. In a multi-center study, probable Alzheimer disease (AD, $n=18)$ and probable dementia with Lewy bodies $(D L B, n=3 I)$ were studied and standardized by the calibration phantom method.

Results. Linear regression equations between LE and ME collimators were obtained for the phantom study in all institutions. When the H/M ratio with a LE collimator was corrected based upon the calibration phantom, the corrected values were comparable to those obtained using ME collimators. The standard database also exhibited a normal distribution after standardization as determined by skewness and goodness-of-fit test. A mixture of the 
populations by LE and ME collimators showed significant separation of AD and DLB groups ( $F$ ratio $=24.9$ for the late $H / M$ ), but the corrected values resulted in higher $F$ ratios for both early and late $H / M$ (F ratio= 34.9 for the late $H / M)$.

Conclusion. Standardization of $\mathrm{H} / \mathrm{M}$ ratios by the heart-chest calibration phantom method is feasible among different collimator types. This method could be practically used for multicenter comparison of $\mathrm{H} / \mathrm{M}$ ratios.

Key Words: ${ }^{123}$ I-metaiodobenzylguanidine; heart-to-mediastinum ratio; calibration phantom; Lewy-body disease; standardization

\section{Introduction}

${ }^{123}$ I-metaiodobenzylguanidine (MIBG) has been used extensively in fields of cardiology and neurology as a unique imaging method to evaluate sympathetic nerve activity and its integrity [I-7]. Inter-institutional differences in MIBG quantification using the heart-tomediastinum ratio $(\mathrm{H} / \mathrm{M})$ have hampered multi-center comparison of the $\mathrm{H} / \mathrm{M}$ ratio, and single-center results could not easily be extrapolated to other hospitals [8]. Since the difference in collimator type has been investigated and found to be one of the major confounders of the results, a practical standardization method for quantification has been sought [9-I2].

In neurological studies, patients with Parkinson disease and dementia with Lewy bodies (DLB), which is categorized as Lewy-body disease demonstrated significantly lower $\mathrm{H} /$ $M$ ratios both in early and late imaging [4-7]. Since the patients with Lewy-body disease showed lower $\mathrm{H} / \mathrm{M}$ values compared with those of normal populations, we used this patient group for a pilot study of the procedure for correction of $\mathrm{H} / \mathrm{M}$ ratios. In addition, since a multi-center study including DLB and MIBG imaging has started in Japan, an appropriate correction method was indispensable to overcome the variation of normal values among participating hospitals. The aim of our study was to standardize the MIBG H/M ratio using a calibration method and examine the effect of this correction on pilot studies. The method was applied to two settings: (I) MIBG normal databases created by a Japanese Society of Nuclear Medicine (JSNM) working group and (2) the multicenter study examining discrimination of DLB from Alzheimer's disease (AD) using MIBG.

\section{Materials and methods}

123I-MIBG study

MIBG studies were performed as a research procedure, with images acquired at 20 minutes and 180 minutes after intravenous injection of II I MBq of ${ }^{123}$ I-MIBG. Early and late images were obtained by both anterior planar image and single-photon emission tomography (SPECT). Planar images were obtained in $256 \times 256$ matrices, and acquisition time was 180 seconds. The energy for ${ }^{123}$ | was centered at $159 \mathrm{keV}$ with a window of $20 \%$. Only the planar anterior image was used in this study, although the SPECT image was routinely used for image interpretation.

\section{Phantom design}

The calibration phantom for ${ }^{123}$ |-MIBG was used as previously described [I I, I3]. Briefly, the phantom was made for a planar-image acquisition and consisted of multiple slices of heart, lung, mediastinum and liver parts. All the parts were filled with radioisotope as a 
single compartment, so that no adjustment of ${ }^{123}$ concentration in each organ part was required. In this study, two types of phantom were used to calculate 4 sets of $\mathrm{H} / \mathrm{M}$ ratios. True $\mathrm{H} / \mathrm{M}$ ratios were mathematically calculated in these models, assuming the linear attenuation coefficient of ${ }^{123} \mathrm{I}$ for water as $0.147 / \mathrm{cm}$. The theoretical values were 2.60 and 3.50 for one type and 1.55 and 1.80 for the other type using anterior and posterior views [II].The calibration phantom and a typical image are shown in Figure I. The H/M values using ME collimators were used as the reference for the study.

\section{Calculation of $\mathrm{H} / \mathrm{M}$ ratios}

In the clinical and phantom studies, a semi-automatic algorithm for determining mediastinal region of interest (ROI) was used [ I4]. After manually setting a circular ROI on the center of the heart, a mediastinal rectangular ROI was automatically determined on the upper third of the mediastinum. The width of the mediastinal ROI was one tenth the width of the chest.

Participating hospitals for the phantom study

The phantom acquisitions on each camera were performed with the two different collimators. Ten hospitals participated in the phantom study (see acknowledgment section of participating hospitals). The anterior images of the phantom were obtained with both lowenergy (LE) collimator and either a low-medium-energy (LME) or medium-energy (ME) collimators. The phantom was filled with water, including II IMBq of ${ }^{123}$ I-MIBG. The anterior and posterior images were obtained using $256 \times 256$ matrices for 3 minutes. All the data were transferred to Kanazawa University with a Digital Imaging and Communication in Medicine (DICOM) format for evaluating the correction method.

Eleven gamma-camera systems were used for MIBG studies in ten institutions (Table I). The $\mathrm{H} / \mathrm{M}$ ratio was corrected using a regression equation between LE- and ME-type collimators which was determined by the calibration phantom. Since four points were given by the phantom experiment, a linear regression equation of $(y-I)=$ slope* $^{*}(x-I)$ was also calculated by the least square fitting method. As this regression line passes through $(x, y)=(I$, $\mathrm{I}$ ), the corrected and uncorrected $\mathrm{H} / \mathrm{M}$ ratios always become I when cardiac count is equal to mediastinal count. The $H / M$ values from the ME collimator were used as the standards, since the ME-collimator type is known to provide a minimal variation [I2].

\section{JSNM working group database}

The JSNM working group for standardization of myocardial SPECT has accumulated MIBG normal databases of early and late anterior images and SPECT [15, 16]. The selection criteria of the normal databases involved patients who had none of the following conditions: electrocardiographic evidence of myocardial ischemia, baseline cardiac diseases (including coronary artery disease, valvular heart disease and severe arrhythmia), history of heart failure, severe liver dysfunction, renal dysfunction, history of heart failure, hypertension with medication, diabetes with medication and dyslipidemia with medication. Those who had coronary stenosis of $<50 \%$ and those who had no indications for coronary angiography could be included if the criteria above were satisfied. The databases consisted of 37 (16 men and 21 women) using the LE collimator and 25 ( 12 men and I 3 women) with the LME or ME collimator. The average age was $57 \pm 19$ years old. The correction of the $\mathrm{H} / \mathrm{M}$ ratio with the LE collimator using the calibration phantom method was examined.

\section{Multi-center study}

To evaluate the effect of standardization in multiple institutions, patients suspected of having AD and DLB were selected for this comparative study. The diagnosis was 
independently performed by neurology specialists. General physical and neurologic examinations, neuropsychological evaluations and appropriate laboratory studies were conducted to confirm the accuracy of the clinical diagnosis. The diagnosis of AD was made in accordance with the National Institute of Neurological and Communicative Disorders and Stroke and the Alzheimer's Disease and Related Disorders Association [17]. The diagnosis of DLB was made in accordance with DLB consensus criteria [18]. In probable AD patients $(n=18$, age $75 \pm 8$ years), I I patients were studied using an LE collimator and 7 using an LME collimator. In probable DLB patients ( $n=31$, age $79 \pm 5$ years), 16 patients were studied using an LE collimator and I 5 using an LME collimator. The standardized $\mathrm{H} / \mathrm{M}$ ratio was calculated by the calibration phantom method.

\section{Institutional Review Board}

To accumulate the patient clinical data and the results of nuclear imaging, all Institutional Review Boards or Ethical Committees approved the study for conducting the MIBG research and transferring the DICOM image data to the core laboratory. Written informed consents were obtained from all patients. The use of JSNM databases was approved by the working group.

\section{Statistics}

All data were expressed as an average \pm standard deviation (SD). The values were compared with analysis of variances (ANOVA) and T test using statistical software (JMP 8.0, SAS institute, Inc). The regression line of $\mathrm{H} / \mathrm{M}$ ratios between measurements was calculated by the least square fitting method. Regarding the asymmetry of distribution, median, quartile points and skewness were used.A positive skew indicates that right tail is longer, and mass of the distribution is concentrated on the lower values. The fitness to normal distribution was examined by a normal quantile plot and a Shapiro-Wilk test. A P value $<0.05$ was considered significant.

\section{Results}

\section{Phantom study}

$\mathrm{H} / \mathrm{M}$ ratios calculated with LE and ME collimators are plotted in Figure 2. In all hospitals, the $\mathrm{H} / \mathrm{M}$ ratio calculated with LE collimators showed lower values than those with ME collimators, and a linear relationship between both methods was observed. The linear regression line between methods with LE and ME collimators differed slightly based on camera types used in each hospital. However, the average regression line was $y=0.460+$ $0.646 x$, where $x$ and $y$ were $H / M$ ratios calculated by ME and LE collimators, respectively, whereas when it was calculated to pass through the point $(I, I)$, the line was $y-I=0.7 I 9$ $(x-I)$. In each institution the corrected $\mathrm{H} / \mathrm{M}$ ratios from LE to ME-comparable values were calculated by the individual institutional regression line, not by the average regression line.

\section{JSNM working group database}

The distribution histogram of the normal database is shown in Fig. 3. The average H/M ratio from the LE collimator was significantly lower than that from the LME/ME collimators $(p<0.000 \mathrm{I}$ for early and late $\mathrm{H} / \mathrm{M}$ ratios). When these two groups were mixed (Fig. 3B), the distribution became asymmetric showing low median values. After the correction of $H / M$ ratios with the LE collimator using the phantom method, the histogram shape became symmetrical in comparison to that of normal distribution. The skewness improved from 0.89 to 0.02 for the early $\mathrm{H} / \mathrm{M}$ ratio, and from $\mathrm{I} .28$ to 0.44 for the late $\mathrm{H} / \mathrm{M}$ ratio. When the Shapiro-Wilk goodness-of-fit test was applied, the uncorrected $\mathrm{H} / \mathrm{M}$ ratios were not 
normally distributed ( $p=0.014$ and 0.0003 for the early and late $H / M$ ratios). In contrast the corrected $\mathrm{H} / \mathrm{M}$ ratios showed normal distribution ( $\mathrm{p}=0.97$ and 0.42 for the early and late $\mathrm{H} /$ $M$ ratios). The average values after standardization did not differ significantly from those with LME/ME collimators $(3.05 \pm 0.42$ and $3.01 \pm 0.53$, respectively, $p=n$. s.).

\section{Multi-center study}

Patients with probable DLB showed lower H/M ratios than those with probable AD regardless of collimator selection (Table 2). Mixed $\mathrm{H} / \mathrm{M}$ ratios with $\mathrm{LE}$ and $\mathrm{LME}$ collimators showed significant differences between the AD and DLB groups ( $F$ ratio=20.4 and 24.9 for early and late $H / M$ ratios). However, when the $H / M$ ratio was corrected by the calibration phantom method, the $F$ ratio increased to 31.7 and 34.9 for the early and late $H / M$ ratios, respectively $(\mathrm{p}<0.000 \mathrm{I}$ for all). When the skewness of the distribution of the $\mathrm{H} / \mathrm{M}$ ratio was anlayzed in the probable AD patients, it improved from 0.60 to -0.003 in the early $\mathrm{H} / \mathrm{M}$ ratio and from 0.87 to 0.02 in the delayed $\mathrm{H} / \mathrm{M}$ ratio after standardization. The changes of skewness implied more even or symmetric distributions after standardization. The effect of correction was more significant in the probable AD group, as the DLB patients showed significantly lower values near I.0.

\section{Discussion}

The need for standardization of MIBG quantification has been discussed particularly from the viewpoint of inter-institutional comparison and multi-center studies. Although several ideas have been proposed, practical solutions acceptable to all institutions have not been proposed $[8,19]$. The present study demonstrated that the calibration phantom method could provide a similar linear relationship between LE and LME/ME collimators in 10 institutions and that it could be applied to different camera-collimator systems. After mixing $\mathrm{H} / \mathrm{M}$ ratios by different camera-collimator settings, the standardization provided more uniform distribution as determined by skewness and goodness-of-fit test to the normal distribution.

${ }^{123}$ I-MIBG imaging has been extensively used in patients with ischemic heart disease and heart failure [I, 2, 20-22]. MIBG imaging was approved by the Japanese Circulation Society in its guidelines, and the indications were classified into Class I (evidence and/or general agreement) and Class lla (weight of evidence is in favor) [23]. In most of the studies, the quantification of cardiac MIBG activity was based on a simple ROI setting on the heart and mediastinum. In the recent ADMIRE-HF study, patients with a low $\mathrm{H} / \mathrm{M}$ ratio showed a significantly higher incidence of serious cardiac events including progression of heart failure, serious arrhythmia and cardiac death [2I].

We selected patient groups with Lewy bodies as a pilot study, since they have pathological background of decreased MIBG uptake [5, 6]. The myocardial MIBG defects reflected a pathological progression of the disease process involving extensive autonomic involvement, not restricted to the brain. In this study, AD patients showed a significantly higher $\mathrm{H} / \mathrm{M}$ ratio than that of the DLB patients, but these values were lower than that of the normal $\mathrm{H} / \mathrm{M}$ ratio calculated with JSNM normal databases. Since the age of AD patients was higher than that of the JSNM normal patients, the effect of age-related change (average age, $75 \pm 8$ vs. $57 \pm 19$ years old) and complicated diseases might partly explain the difference in the $\mathrm{H} / \mathrm{M}$ ratios [24].

The effects of collimator selection have been evaluated, and a number of studies have indicated that $\mathrm{H} / \mathrm{M}$ ratios with the $\mathrm{LE}$ collimator showed a lower value compared to that with the ME collimator [9-12, 19].Verberne et al. found that the collimator type was one of the independent predictors of late $\mathrm{H} / \mathrm{M}$ for subjects with a late $\mathrm{H} / \mathrm{M}$ I.4- 2.0. Normal values 
assembled by the different types of collimators showed significant differences in Japanese standard MIBG databases: $2.39 \pm 0.2 \mathrm{I}$ and $2.49 \pm 0.25$ for early and late imaging using an LE collimator, and $2.76 \pm 0.3 \mathrm{I}$ and $3.0 \mathrm{I} \pm 0.35$ using an ME collimator, respectively $(p<0.000 \mathrm{I}$ for both early and late $\mathrm{H} / \mathrm{M}$ ratios) $[15,16]$. While several methods to overcome collimator differences have been proposed [ II, 25], a European group recommended the use of ME-type collimators as a standard procedure based on a phantom study [10]. However, we examined the calibration phantom approach in this study, since the idea of cross-calibration among nuclear medicine systems was supposed to be straight-forward and applicable to any institution.

After the standardization of normal databases, the mixture of $\mathrm{H} / \mathrm{M}$ ratios by LE and ME collimators became a normal distribution. The use of single normal value would be convenient for clinical practice. In patients with abnormal MIBG studies, a mixture of $H / M$ values from cameras with the LE, LME and ME types of collimators may have resulted in potentially low diagnostic ability. Since the H/M ratio was apparently low in patients with Lewy-body disease, separation between Lewy-body disease and near-normal MIBG patients was only slightly affected by the correction in this study. However, in patients with heart failure and in non-heart failure groups, the $\mathrm{H} / \mathrm{M}$ ratios may not have been clearly separated, showing continuous distributions with an overlapped zone. Since the separation of two groups depends on their average and deviation, whether diagnostic accuracy was enhanced by the standardization should be carefully interpreted. We presumed a mixture of $\mathrm{H} / \mathrm{M}$ ratios using two types of collimators in this study. Although identical acquisition parameters should be decided in the research purpose, differences in collimator designs still existed depending on camera venders even with similar collimator types, such as LEHR, LEGP and other special LE types covering ${ }^{123}$ | energy $[9,10]$.

Some limitations are described. The standardization proposed in this study was not to create theoretically true or scatter- and septal-penetration-free images. It only provided an ME-collimator comparable $\mathrm{H} / \mathrm{M}$ value, since the use of the ME collimator minimized the septal penetration among currently available collimators [12]. Although the recommendation to use ME-type collimators is a reasonable approach, many institutions continue to use LEtype collimators because of collimator availability and the inconvenience of changing collimators from study to study in daily practice. The calibration phantom was created for planar studies and could not be used for SPECT studies. However, to calibrate anterior images from septal penetration and partly from Compton scatter, the use of phantom calibration seemed to be a practical method. Although $\mathrm{H} / \mathrm{M}$ ratios from ME and LME collimators showed similar values in our camera-collimator setting [I I], those from other camera venders may differ significantly. The difference in LME and ME collimators, if present, would be also confirmed by the calibration phantom.

\section{Conclusion}

123I-MIBG H/M ratios measured by an LE collimator were corrected to ME collimatorcomparable values using a calibration phantom. In normal databases the correction method provided comparable values between the use of LE- and LME/ME-type collimators, resulting in normal distribution of $H / M$ ratios. Although a mixture of $L E$ and $M E$ collimator data in $A D$ and DLB groups could be separated even before correction, this correction method provided a better separation of the two groups. When gathering MIBG studies as a multi-center study, the calibration phantom method can be readily applied in all hospitals, and it would potentially enhance diagnostic ability. 
Table I. Scinticamera venders and collimator types in 10 institutions

\begin{tabular}{ccccc|}
\hline institution & camera vender & LE types & ME types & Routine study \\
\hline A & Toshiba & LE HR & LME GP & ME \\
BI* & Shimadzu & LE GP & ME GP & LE \\
B2* & Siemens & LE HR & ME & LE \\
C & Siemens & LE HR & ME & LE \\
D & Toshiba & LE HR & LME GP & ME \\
E & Philips & LE HR & ME GAP & LE \\
F & Siemens & LE HR & LME GP & LE \\
G & Siemens & LE HR & LME GP & ME \\
H & GE & ELE GP & ME & LE \\
I & Siemens & LE HR & ME & LE \\
J & Shimadzu & LE HR & ME & LE \\
\hline
\end{tabular}

ME, medium energy; LE, low energy; HR, high resolution; GP, general purpose; ELE, extended low-energy; LME, low-medium-energy; GAP, general all purpose; Nomenclatures of the collimator type depending on the venders; ${ }^{*}$ Institution B uses two scinticameras.

Table 2. Comparison of $\mathrm{H} / \mathrm{M}$ ratios between Alzheimer disease and dementia with Lewy bodies
AD
DLB
ANOVA
$\mathrm{n}$ mean $\pm \mathrm{SD}$
$\mathrm{n}$ mean $\pm \mathrm{SD} \quad \mathrm{F}$ ratio
P

\section{Early H/M}

No correction

$\mathrm{H} / \mathrm{M}$ with LE

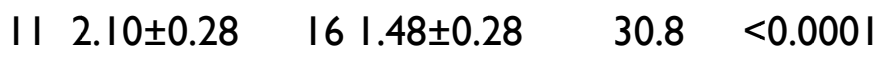

H/M with LME

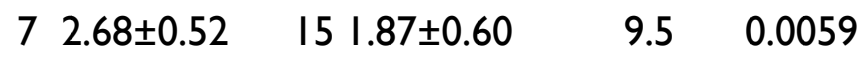

Mixed $\mathrm{H} / \mathrm{M}$ with LE and LME

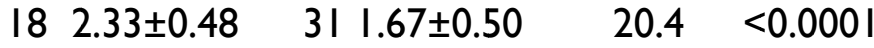

Standardized HIM with LE and ME

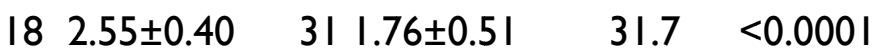

\section{Late $H / M$}

No correction

$H / M$ with LE

$\begin{array}{lllll}\text { II } & 2.09 \pm 0.26 \quad 16 \quad 1.31 \pm 0.31 \quad 45.8 \quad<0.0001\end{array}$

H/M with LME

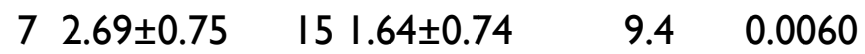

Mixed $\mathrm{H} / \mathrm{M}$ with LE and LME

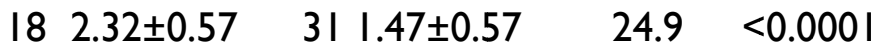

Standardized $H / M$ with $L E$ and $M E$

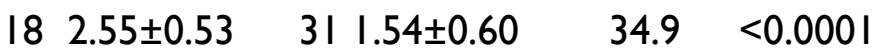

AD, Alzheimer disease; DLB, Dementia with Lewy bodies 


\section{Disclosure Statement}

Authors declare no conflict of interest in this study.

\section{Acknowledgment}

We thank the physicians and technologists who contributed to the phantom studies and accumulation of the data. This work was supported partly by Grants-in-Aid for Scientific Research in Japan (No. 2259|320, P. I. Kenichi Nakajima).

\section{Figure Legends}
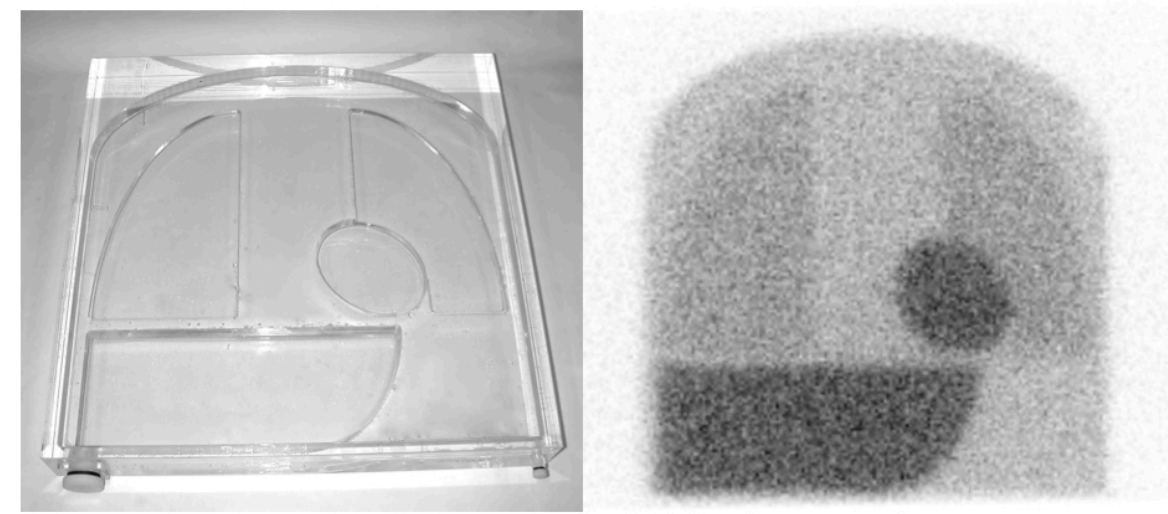

\section{Figure I}

123/-MIBG calibration phantom (left) and a typical scintigraphic image (right).

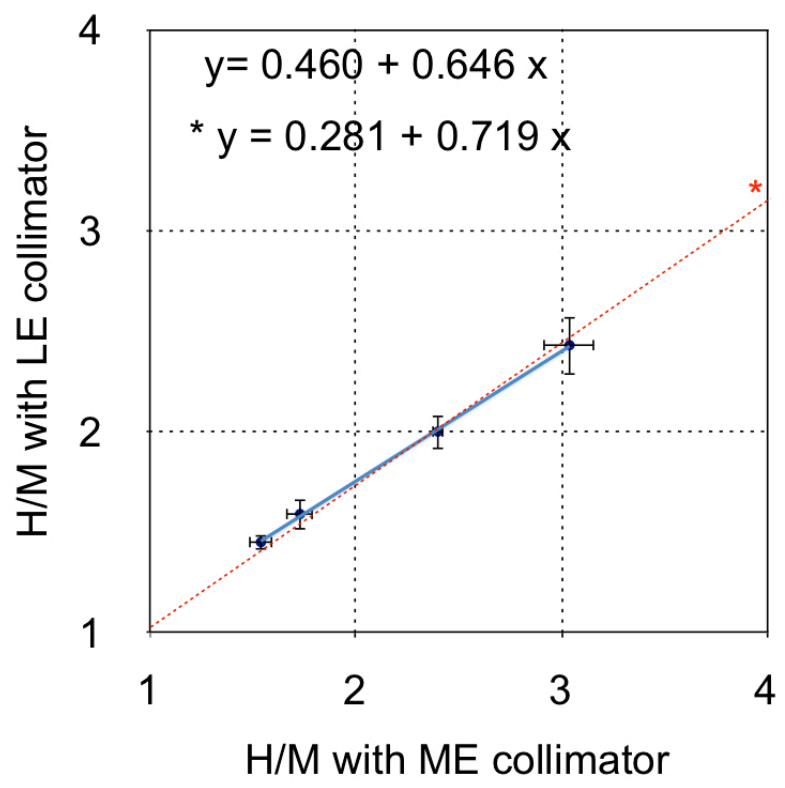

\section{Figure 2}

Relationship of $\mathrm{H} / \mathrm{M}$ ratios obtained from 10 hospitals (I I camera types). $\mathrm{H} / \mathrm{M}$ ratios from ME and LE collimators showed a linear relationship. 
A No correction

LE LME or ME

Early

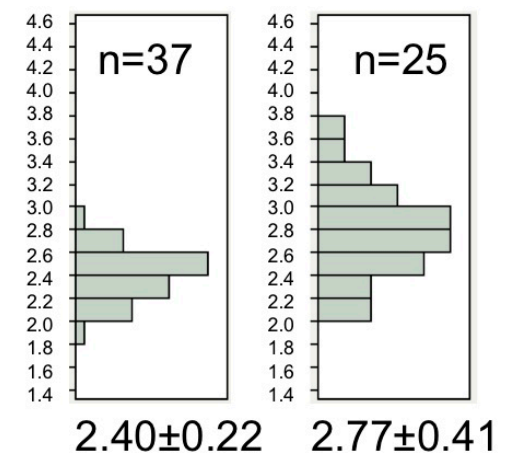

Late

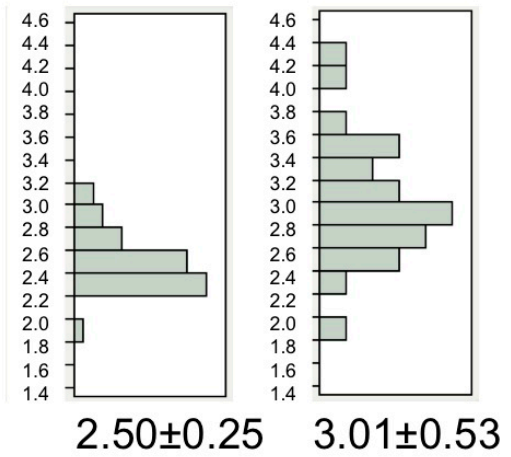

B Mixed (No correction)
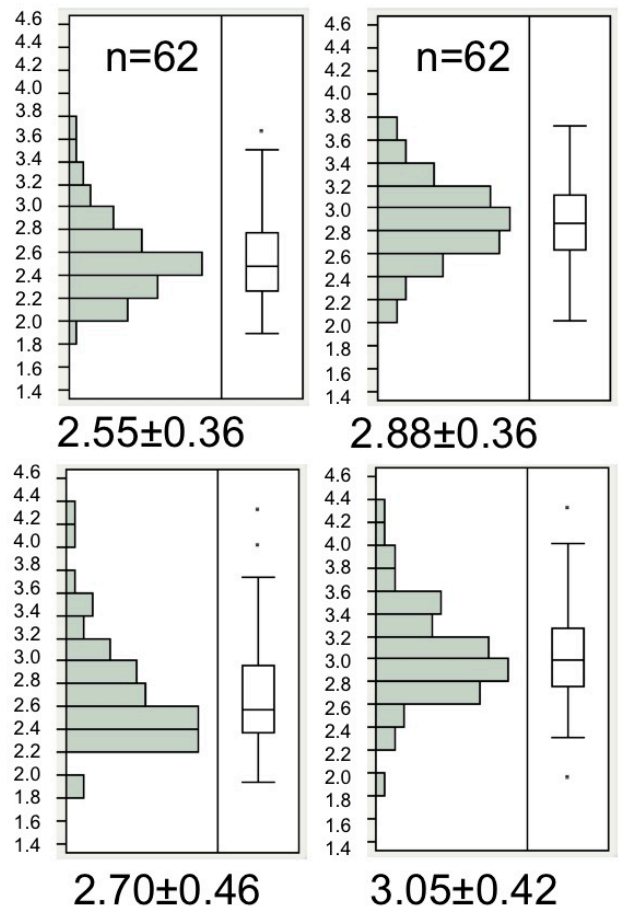

\section{Figure 3}

Histograms of normal patients from JSNM working group databases. (A) Early and late $H / M$ ratios without correction. (B) Distribution of $H / M$ ratios when LE and LME/ME groups were mixed. (C) Distribution of $\mathrm{H} / \mathrm{M}$ ratios when those from LE collimator are corrected by the calibration phantom method. After the standardization, the distribution became normal. The skewness was 0.89 and $I .28$ in B, and 0.02 and 0.44 in $C$ for the early and late H/M ratios, respectively. The outlier box plot indicates median and 25 and $75 \%$ quartiles.

\section{References}

I. Merlet P, Valette H, Dubois-Rande JL, Moyse D, Duboc D, Dove P, et al. Prognostic value of cardiac metaiodobenzylguanidine imaging in patients with heart failure. J Nucl Med. 1992;33:47 I-7.

2. Matsunari I, Schricke U, Bengel FM, Haase HU, Barthel P, Schmidt G, et al. Extent of cardiac sympathetic neuronal damage is determined by the area of ischemia in patients with acute coronary syndromes. Circulation. 2000; I 0 I:2579-85.

3. Jacobson AF, Lombard J, Banerjee G, Camici PG. I23I-mIBG scintigraphy to predict risk for adverse cardiac outcomes in heart failure patients: design of two prospective multicenter international trials. J Nucl Cardiol. 2009; I6:I I3-2I.

4. Yoshita M, Taki J,Yokoyama K, NoguchiShinohara M, Matsumoto Y, Nakajima K, et al.Value of I 23I-MIBG radioactivity in the differential diagnosis of DLB from AD. Neurology. 2006;66: I850-4.

5. Nakajima K, Yoshita M, Matsuo S, Taki J, Kinuya S. lodine- I 23-MIBG sympathetic imaging in Lewy-body diseases and related movement disorders. Q J Nucl Med Mol Imaging. 2008;52:378-87. 
6. Estorch M, Camacho V, Paredes P, Rivera E, Rodriguez-Revuelto A, Flotats A, et al. Cardiac (I23)Imetaiodobenzylguanidine imaging allows early identification of dementia with Lewy bodies during life. Eur J Nucl Med Mol Imaging. 2008;35: |636-4I. 7. Treglia G, Cason E, Giordano A. Diagnostic performance of myocardial innervation imaging using MIBG scintigraphy in differential diagnosis between dementia with Lewy bodies and other dementias: A systematic review and a meta-analysis. J Neuroimaging. 2010 [Epub ahead of print].

8. Verberne HJ, Habraken JB, van Eck-Smit BL, Agostini D, Jacobson AF.Variations in I23Imetaiodobenzylguanidine (MIBG) late heart mediastinal ratios in chronic heart failure: a need for standardisation and validation. Eur J Nucl Med Mol Imaging. 2008;35:547-53.

9. Inoue Y, Suzuki A, Shirouzu I, Machida T, Yoshizawa Y,Akita F, et al. Effect of collimator choice on quantitative assessment of cardiac iodine 123 MIBG uptake. J Nucl Cardiol. 2003; 10:623-32.

10. Verberne HJ, Feenstra C, de Jong WM, Somsen GA, van Eck-Smit BL, Busemann Sokole E. Influence of collimator choice and simulated clinical conditions on I23I-MIBG heart/mediastinum ratios: a phantom study. Eur J Nucl Med Mol Imaging. 2005;32:1100-7.

I I. Nakajima K, Matsubara K, Ishikawa T, Motomura N, Maeda R,Akhter N, et al. Correction of iodine- I23-labeled meta-iodobenzylguanidine uptake with multi-window methods for standardization of the heart-to-mediastinum ratio. J Nucl Cardiol. 2007; |4:843-5।.

12. Flotats A, Carrio I, Agostini D, Le Guludec D, Marcassa C, Schafers M, et al. Proposal for standardization of I23I-metaiodobenzylguanidine (MIBG) cardiac sympathetic imaging by the EANM Cardiovascular Committee and the European Council of Nuclear Cardiology. Eur J Nucl Med Mol Imaging. 2010;37:1802-12.

13. Matsuo S, Nakajima K, Okuda K, Kawano M, Ishikawa T, Hosoya T, et al. Standardization of the heart-to-mediastinum ratio of $|23|$-labelledmetaiodobenzylguanidine uptake using the dual energy window method: feasibility of correction with different camera-collimator combinations. Eur J Nucl Med Mol Imaging. 2009;36:560-6.

14. Okuda K, Nakajima K, Hosoya T, Ishikawa T, Konishi T, Matsubara K, et al. Semi-automated algorithm for calculating heart-to-mediastinum ratio in cardiac lodine- 123 MIBG imaging. J Nucl Cardiol. 20II;। 8:82-9.

I5. Matsuo S, Nakajima K, Yamashina S, Sakata $\mathrm{K}$, Momose M, Hashimoto J, et al. Characterization of Japanese standards for myocardial sympathetic and metabolic imaging in comparison with perfusion imaging. Ann Nucl Med. 2009;23:5I 7-22.

16. Nakajima K. Normal values for nuclear cardiology: Japanese databases for myocardial perfusion, fatty acid and sympathetic imaging and left ventricular function. Ann Nucl Med. 20 I0;24:I25-35. 17. McKhann G, Drachman D, Folstein M, Katzman R, Price D, Stadlan EM. Clinical diagnosis of
Alzheimer's disease: report of the NINCDS-ADRDA Work Group under the auspices of Department of Health and Human Services Task Force on Alzheimer's Disease. Neurology. 1984;34:939-44. I8. McKeith IG. Consensus guidelines for the clinical and pathologic diagnosis of dementia with Lewy bodies (DLB): report of the Consortium on DLB International Workshop. J Alzheimers Dis. 2006;9:417-23.

19. Yamashina S, Yamazaki J. Role of MIBG myocardial scintigraphy in the assessment of heart failure: the need to establish evidence. Eur J Nucl Med Mol Imaging. 2004;3 I: / 353-5.

20. Merlet P, Hittinger L, Dubois-Rande JL, Castaigne A. Myocardial adrenergic dysinnervation in dilated cardiomyopathy: cornerstone or epiphenomenon? J Nucl Med. 2002;43:536-9. 21. Jacobson AF, Senior R, Cerqueira MD, Wong ND, Thomas GS, Lopez VA, et al. Myocardial iodine- I 23 meta-iodobenzylguanidine imaging and cardiac events in heart failure. Results of the prospective ADMIRE-HF (AdreView Myocardial Imaging for Risk Evaluation in Heart Failure) study. J Am Coll Cardiol. 2010;55:22 I2-2I.

22. Agostini D, Verberne HJ, Burchert W, Knuuti J, Povinec P, Sambuceti G, et al. I-I23-mIBG myocardial imaging for assessment of risk for a major cardiac event in heart failure patients: insights from a retrospective European multicenter study. Eur J Nucl Med Mol Imaging. 2008;35:535-46.

23. Tamaki N. Guidelines for Clinical Use of Cardiac Nuclear Medicine (JCS 2005). Circ J. 2005;69 Suppl. IV I I25-202 (Revision on the web site 20 I0). 24. Sakata K, Shirotani M, Yoshida H, Kurata C. Physiological fluctuation of the human left ventricle sympathetic nervous system assessed by iodine-123MIBG.J Nucl Med. 1998;39:I667-7I.

25. Chen J, Garcia EV, Galt JR, Folks RD, Carrio I. Optimized acquisition and processing protocols for I- 123 cardiac SPECT imaging. J Nucl Cardiol. 2006; | 3:25|-60. 